\section{Familial Medullary Carcinoma of the Thyroid}

Medullary carcinoma of the thyroid is a tumour of the parafollicular or $\mathrm{C}$ cells, which produce calcitonin. It tends to occur in families, and is associated with phaeochromocytomas, parathyroid hyperplasia or adenomas, and occasionally with Cushing's syndrome. ${ }^{2}$ It has been classified ${ }^{3}$ as the multiple-endocrine-adenomatosis syndrome, type II, as distinct from the type I syndrome characterized by pituitary, parathyroid, pancreatic islet cell, and adrenal adenomas. Some patients with medullary carcinoma of the thyroid have a combination of multiple buccal and orbital neuromas, an unusual facies with thick lips, a marfanoid habitus, a proximal myopathy, and megacolon. ${ }^{4}$ Most cases with multiple neuromas have been sporadic, and this could prove to be an entity distinct from the multiple-endocrine-adenomatosis syndrome type II.

It should now be standard practice to investigate the families of patients with the medullary carcinoma of the thyroid syndrome: the disease is inherited as an autosomal dominant with a high degree of penetrance. ${ }^{5}$ Clinical evidence of the syndrome may be detected and may warrant surgical intervention. Biochemical tests should be performed to exclude the presence of a phaeochromocytoma before operating on the thyroid. In the familial syndrome bilateral phaeochromocytomas may be asymptomatic and appear to be non-functioning, but under conditions of stress such as a surgical operation a catastrophic release of catecholamines may occur. Routine screening tests such as the urinary excretion of hydroxymethoxymandelic acid (HMMA) may be negative, since small tumours have a faster turnover rate of catecholamines ${ }^{6}$ and may produce an increased excretion of free catecholamines in the urine without a corresponding increase in HMMA. Adrenal echograms or arteriograms or estimation of the catecholamine content of specimens obtained by venous catheterization may also provide evidence for the presence of the adrenal tumour.

Parathyroid hyperplasia, or less often adenoma formation, is part of the syndrome but does not always cause features of hyperparathyroidism with hypercalcaemia. It is uncertain whether the parathyroid hyperplasia is a response to the increased circulating calcitonin levels or to a genetically associated abnormality. Raised parathormone levels in the absence of abnormalities of circulating calcitonin were found in one kindred, ${ }^{7}$ which gives support to the latter possibility.

In the absence of clinically detectable thyroid enlargement radioisotope scanning of the thyroid may be helpful; calcification in the tumour or secondary deposits has been described. ${ }^{8}$ The most accurate screening test for the presence of medullary carcinoma is measurement of calcitonin in the peripheral circulation. ${ }^{9} \mathrm{~A}$ raised serum level of calcitonin in a patient with the familial syndrome with or without mucosal neuromas is an indication for total thyroidectomy after careful exclusion of a phaeochromocytoma. In a small proportion of cases the basal calcitonin level is normal, but a test using a calcium infusion to stimulate calcitonin secretion may produce an abnormality. In patients at risk who have normal basal calcitonin levels and a normal response to calcium it is probably wise to perform annual calcium infusion tests to detect disease at an early stage, and indeed two sisters at risk for hereditary medullary carcinoma who were tested in this way were found to have C-cell hyperplasia in the middle and upper portions of the lateral lobes of the thyroid. ${ }^{10}$ At that stage the process may be preinvasive, so that total thyroidectomy should prove to be curative. The increased numbers of $\mathrm{C}$-cells in the abnormal glands may be shown by routine haematoxylin and eosin staining as well as by more elaborate immunofluorescent or immunoenzymatic techniques. The multifocal nature of the bilateral hyperplasia correlates with the multicentric origin of the medullary carcinoma.

Calcitonin measurements are preferable to serum histaminase estimation ${ }^{11}$ in the diagnosis of medullary carcinoma of the thyroid. When provocative stimuli are required calcium infusions seem to be more reliable than glucagon or gastrin, which also stimulate the release of calcitonin. Furthermore glucagon is known to release catecholamines, which would prove dangerous in a patient with a phaeochromocytoma.

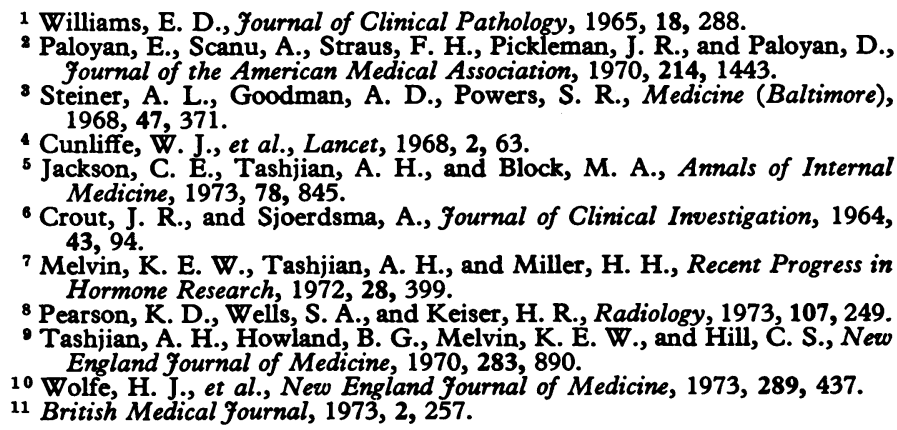

\section{Treatment of Genital Herpes}

Effective chemotherapy for virus infections is a challenge to biochemical ingenuity, since the complexity of the union between host and virus makes it difficult to inhibit the multiplication of the virus without at the same time damaging the host cells. One of the more elegant solutions is to incorporate a light-sensitive compound into the virus and then to irradiate the cells. The virus is made hypersensitive to light and is inactivated by the illumination. It is nearly 10 years since Wallis and Melnick ${ }^{1}$ suggested that this principle might be harnessed to the treatment of herpetic lesions in man, using a heterotricyclic dye such as acridine orange. A controlled trial using neutral red gave some success, ${ }^{2}$ and recently the method has been used in herpes vulvitis by Friedrich, ${ }^{3}$ Lefebure and McNellis, ${ }^{4}$ and a group including Wallis and Melnick themselves. ${ }^{5}$ This last study concerned 49 women aged 8 to 52 suffering from herpes genitalis. Twenty cases were described as primary and 28 as recurrent on serological grounds, the remaining one being unclassified. The duration of symptoms before treatment was in most cases less than 7 days. Local application of $0.1 \%$ proflavine was followed by irradiation with a 150-watt incandescent or fluorescent light for 10 minutes. The patients reported back in 24 hours and the results were assessed. Over half reported relief of pain within 2 hours, and most within 24 hours. Only 6 reported the degree of pain relief as poor, and even when the observed appearances were not much improved there was considerable subjective improvement. Kaufman et al. suggest that for virological reasons it would have been best to have given another treatment after 18 hours and that the use of monochromatic light may prove to be an advantage.

The evaluation of these results is difficult. Herpes genitalis is an unpleasant and troublesome condition, therapeutically somewhat intractable, and like all herpes infections apt to recur. It could be argued that any alleviation caused by the 